\title{
SEMINARIO DE PEQUEÑAS Y MEDIANAS EMPRESAS
}

\author{
Luis Bortesi Longhi \\ Docente de la Facultad de Ciencias Contables \\ Jorge Calampa Guriz, Soraya \\ Castro Herrera, Carmen Córdova Moreno, \\ Julián García Céspedes, Robert Miranda Castillo, \\ Bernardo Sánchez Barraza, Lily. Trigos Sánchez \\ Alumnos - Post Grado
}

\begin{abstract}
RESUMEN
Uno de los temas que suscita mucho interés en la investigación para impulsar el desarrollo económico es el de las pymes, porque, según se ha constatado fehacientemente, ese tipo de organizaciones ofrecen algunas ventajas muy particulares frente al emprendimiento que supone la mediana y gran empresa. La flexibilidad y ductilidad de la micro y pequeña empresa es, por lo tanto, una primera ventaja. También aportan la conveniencia y preferencia comparativa de ser capaces de producir más oferta de trabajo con menos inversión, es decir, que marcan un ratio capital/puesto creado mucho más bajo y asequible que el que exige una organización mediana o grande.
\end{abstract}

Por anterior, por la flexibilidad y por requerir menor inversión, la contribución de las pymes al producto bruto interno (PBI) viene a ser muy significativa.

El peso que han llegado a tener las pequeñas empresas a nivel mundial demuestra que esa concepción organizacional configura una fórmula ventajosa universalmente reconocida.

Un aspecto vertebral que atañe directamente al Perú y a la pequeña y microempresa, es el de la formalización puesto que los índices de informalidad de entidades productivas que no están registradas, que no tienen licencra, que no cuentan con los respectivos libros de contabilidad y que no reconocen los beneficios socrales, arrojan porcentajes impresionantemente altos en el Perú. Por eso, además de todas las virtudes señaladas líneas arriba, está la virtud singular del incentivo a la formalización, que bien podría decirse, a guisa de sinónimo, modernización.

Hay otros múltiples aspectos a los que conviene prestar atención alrededor del tema de las micro y pequeñas empresas en el Perú. Uno de los más importantes de ellos, y seguramente vinculado a la informalidad, es el de la vida efimera en que muchas veces transcurre el destino de las pyme. Así como exısten estadísticas donde se estima un enorme número de nacimientos, así también está presente la precarıedad y la vida corta $e$ inestable que termina en el deceso de la micro organización.

En medio de todo ese escenario anteriormente descrito, se promulga en el Perú la Ley 28015, llamada de Promoción y Formalización de la Micro y Pequeña Empresa (el 3 de julio de 2003), la misma que. según declaraciones del ex Ministro de Trabajo (en el Perú las micro y pequeñas empresas están adscritas al sector trabajo) Juan Sheput no ha tenido éxito; siendo, por lo tanto, imprescindible, dadas las circunstanclas, tener que proceder al perfeccionamiento legal e institucional de lo que se debería conocer como Sistema Nacional de las Micro, Pequeñas y Medianas Empresas.

La puerta más auspiciosa para impulsar a las micro y pequeñas empresas es la de las exportaciones y es en esa dirección que el Tratado De Libre Comercio con los Estados Unidos de América alienta y estimula el proyecto nacional que consagra la primera prioridad a las pymes.

Palabras clave: Sistema, empresa, empleo, organización, micro, evolución, mercado, crecimiento, desarrollo. 


\section{INTRODUCCIÓN}

Durante el segundo semestre del año 2005, la Unidad del Post Grado de la Facultad de Ciencias Contables dela Universidad Nacional Mayor de San Marcos organizó un curso para el segundo ciclo del Doctorado con el título de "Seminario de Pequeñas y Medianas Empresas». Siete doctorandos integran la promoción, los quebajola dirección del doctor Luis Bortesi abordaron la investigación discutiendo, ante todo, si el seminario consideraría a las micro y pequeñas empresas como la primera prioridad para el desarrollo económico del Perú; o, por el contrario, habría que criticar como a una especie de espejismo una perspectiva tan optimista.

La discusión fue ardua especialmente por la riqueza del tema y por la experiencia y formación profesional de todos los participantes.

En conclusión, se acordó que en vista dela multiplicidad de argumentos favorables (los que se mencionan en el párrafo anterior como «resumen"), en las circunstancias actuales las micro y pequeñas empresas en el Perú constituyen una organización potencial con todos los méritos para ocupar una primera prioridad en los programas de desarrollo económico.

Una vez que se hubo adoptado, por unanimidad, la posición de partida, el siguiente paso consistió en identificar los temas más relevantes para investigar la realidad y el destino de las micro y pequeñas empresas. Luego, el procedimiento consistiría en diagnosticar cada tema en su situación actual para proponer estrategias que permitan la consolidación y desarrollo de las pyme.

Los aspectos específicos más destacables que se seleccionaron para la investigación fueron los siguientes:
- Mercado

- Financiamiento

Capacitación

Gestión

Asociación

Aspectos tributarios

Aspectos laborales.

En todos los casos, las propuestas estratégicas para favorecer el desarrollo de las micro y pequeñas empresas, según acuerdo unánime, tendrían que obedecer a tres Líneas Maestras Rectoras, que marcan el propósito final, con alcance de Proyecto Nacional. Tales líneas fueron las siguientes:

- Que las micro empresas deben EvoluCIONAR hacia pequeñas empresas $y$, a su vez, las pequeñas empresas hacia medianas.

- Que los incentivosotorgados deben tener carácter prioritario cuando coadyuven a la DESCENTRALIZACIÓN.

- Que la FORMALIZACIÓn es, concomitantemente, un objetivo nacional prioritario.

La presente publicación contiene principalmente el resumen de las propuestas que sugiere con respecto a los temas aludidos, así como a aspectos generales, la Promoción del Doctorado.

\section{PRINCIPALES CONSIDERACIONES Y PROPUESTAS}

1. Crear por ley, el Sistema Nacional de Promoción y Desarrollo de las Mipyme.

2. Instituir un Organismo Nacional Autónomo Coordinador de las MIPYME inscrito en el pliego Presidencia del Consejo de Ministros que asuma las siguientes responsabilidades: 
3. En primer lugar, investigar a cabalidad y profundidad los parámetros para clasificar a las micro, pequeñas y medianas empresas y en consecuencia proponer elementos de juicio cientificos, de carácter económico, social, étnico y técnico que conlleven a una clasificación clara y justa en cada sector y en cada región.

Naturalmente la clasificación tendrá carácter dinámico.

(Es de remarcarse que en el actualidad concurren solamente dos criterios: el número de personal empleado y la facturación bruta anual, lo que, sin mayor comentario, configura la vigencia de una perspectiva superficial, poco elaborada, burda.)

4. Diseñar estrategias y políticas para las Mipyme, en aspectos de constitución, formalización, competencias gerenciales, desarrollo del capital social, capacitación, transferencia e innovación tecnológica, mercadeo, financiamiento y trámites administrativos.

5. Mantener actualizado el Registro Nacional de Estadística e Informática de las Micro, Pequeñas y Medianas Empresas.

Las empresas inscritas en el registro serán las únicas que gozarán de los beneficios generales de promoción de las Mipyme y esta condición será el resorte para que, motu proprio, procedan a la formalización.

6. Suscribir con el Colegio de Contadores un convenio para difundir un Manual Único Simplificado de Contabilidad.

7. Coordinar y centralizar los programas de promoción y desarrollo de las Mipyme en todos los sectores.

8. Proponer y Vigilar la aplicación de las Normas y Procedimientos de Simplificación Administrativa a nivel de ministerios, regiones y municipios.
9. Direccionar los programas de promoción de las Mipyme en las distintas regiones y localidades del país, incluyendo los municipios.

10. Implementar un Registro Nacional de instituciones de desarrollo para las Mipyme como el objeto de priorizar y seleccionar la asignación de los recursos en programas y proyectos.

11. Centralizar a todos los organismos que actualmente, en los diferentes ministerios e instituciones, tienen competencia sobre las Mipyme.

12. Canalizar todos los recursos nacionales $\mathrm{y}$ de cooperación internacional destinados a la promoción de las Mipyme.

13.Promover el desarrollo de la asociatividad empresarial en primer y segundo nivel, así como en el ámbito territorial, sectorial, regional, nacional e internacional.

14.Incorporar a las universidades al Sistema Nacional de Promoción y Desarrollo de las Mipyme.

Un ámbito particular que debe merecer atención focalizada con el espíritu de promover a las Mipyme es el de la capacitación.

El capital humano será indudablemente el soporte que permita impulsar la prosperidad y consistencia de las micro y pequeñas empresas y debemos reconocer que en nuestra situación actual la calidad de la capacidad empresarial de estos emprendedores es generalmente deficiente y en todos los casos muy heterogénea.

15. Crear un Instituto Central Tecnológico de Capacitación para las Mipyme, perteneciente al Sistema Nacional de Desarrollo y adscrito al Organismo Nacional Autónomo con el objeto de incrementar el nivel de la gestión y la capacidad de competitividad. 
16.La primera perspectiva abordará la formación temprana, en la edad escolar, explicando a los estudiantes, desde niños, las circunstancias para la creación de una pequeña y mediana empresa, incidiendo en la importancia de éstas, su proyección y su sentido práctico.

Todas las nociones básicas de la actividad empresarial productiva serían comprendidas en el plan de estudios privilegiando la práctica antes que la teoría.

Conceptos elementales de: Contabilidad, comercio, práctica bancaria, exportación, organización y procedimientos informáticos deberían integrar el programa de estudios de la asignatura.

17. La segunda perspectiva estaría orientada a la capacitación de los conductores de las pequeñas empresas para instruir y formar pequeños empresarios que se encuentren ya en el ejercicio rudimentario, en los siguientes aspectos:

- Diseño de planes estratégicos

- Gestión de la calidad y la competitividad

- Promoción y acceso a mercados nacionales e internacionales

- Innovación y transferencia de tecnología

- Gestión de información y comunicaciones

- Acceso a fuentes de financiamiento

- Formación de una cultura emprendedora

- Articulación y cooperación inter empresarial

- Comercio exterior.

La financiación del Instituto Central Tecnológico procedería con fondos otorgados por COFIDE, que servirían para sufragar becas seleccionando a los participantes más talentosos $y$ r.j prometedores. La beca en menci itendría carácter reembolsable durante $\mathbf{x}$ etapa posterior de maduración y frut $x$ de la empresa.

Los docentes responsables del institur: serían pertenecientes a la planta orgánica permanente del mismo, profesores universitarios adjuntos, $y$, asimismo, ocuparían la docencia, en condición de invitados, empresarios nacionales $y$ extranjeros de éxito.

Con respecto al Financiamiento de los Proyectos de Inversión, las propuestas son las siguientes:

a) Instituir el Banco de Fomento Sectorial para las Mipyme.

b) El fondo inicial del banco provendría de aportes de COFIDE y de La Corporación Andina de Fomento.

c) Una repartición del banco estaría organizada para formular Proyectos de Inversión, a partir de la elaboración de las ideas que los microempresarios pudieran aportar.

d) Por lo tanto el financiamiento procedería luego del análisis de factibilidad y de calidad estudiado por la oficina de proyectos del banco.

Naturalmente la principal garantía que debería evaluarse para la aprobación del proyecto sería el estudio de mercado.

e) La prioridad en el otorgamiento de los créditos respondería al criterio de descentralización; es decir, no se privilegiarían micro empresas instaladas en Lima.

f) La adjudicación de créditos, tal y como reza la política que inspira a la banca de fomento, se realizaría a través de préstamos «blandos», a mediano y largo plazo, según la naturaleza del 
negocio y con años de gracia y baja tasa de interés.

g) Una obligación inicial del Banco de Fomento Sectorial para las Mipyme sería la de reglamentar criterios más específicos para la atención de solicitudes de proyectos de inversión en cada sector y en cada región.

El Mercado y el comercio nacional e internacional es el elemento clave que habrá de decidir el éxito muy probable de la actividad empresarial.

h) El Organismo Nacional Autónomo contará con un departamento para formular nuevos proyectos de inversión. El eje central de los proyectos lo constituirá el constante monitoreo, estudio e investigación para desbrozar el camino hacia nuevos mercados, ya sea en el ámbito nacional como para promover las exportaciones.

i) Un campo prioritario para el análisis del mercado exterior al que pudiesen acceder las Mipyme lo ofrece, sin duda alguna, el Tratado De Libre Comercio con los Estados Unidos.

j) La aplicación de un «test evaluativo» para medir el nivel de conocimiento sobre los usos de las tecnologías de información y comunicación, a fin de tener una valoración sobre las capacidades de los empresarios como potenciales exportadores.

k) La formación de redes estratégicas para la promoción de alianzas con empresas nacionales, transnacionales $\mathrm{y}$ asociaciones ("clusters») que posibiliten trabajar en un escenario global e interconectado, con acceso permanente a una fuente de información que traspase fronteras a costos reducidos, así como redes de prestación de servicios que coadyuven la competitividad.

1) Promover la creación de una Asociación Latinoamericana de Pymes, organismo internacional que permita concentrar la coordinación de esfuerzos de internacionalización con otras de su misma área o sector, para acceder mancomunadamente a los mercados internacionales.

m) La creación de «Centros Comerciales Virtuales» en los que cada empresa pueda tener un espacio para la promoción de sus productos y servicios a bajo costo. La difusión de las capacidades del comercio electrónico contribuye a disminuir la brecha entre empresas, pese a la gran diferenciación que existe entre sus tamaños.

La necesidad de diseñar y adelantar estrategias colectivas pasa a ser no solamente una posibilidad de desarrollar ventajas competitivas individuales y conjuntas, sino que puede llegar a constituir un requisito básico de sobrevivencia para las Mipyme. Ciertamente, alguna de las estrategias individuales tendrá éxito en la medida en que se complemente con estrategias colectivas. En materia de Asociatividad las propuestas son las siguientes:

a. Organizar conglomerados de textiles, agroindustria, industria, comercio, transporte, turismo y otros, capaces de alcanzar dinámicas de eficiencia colectiva para optimizar la inserción de las empresas en los mercados de manera más competitiva.

b. Promover y sostener el desarrollo de micro y pequeñas empresas compuestas por mujeres empresarias, jóvenes y discapacitados. 
c. Organizar cooperativas con fines de lucro al interior de los centros penitenciarios que promuevan el interés de los reclusos por el trabajo, reconozcan su esfuerzo y creatividad y los estimule a aspirar a una remuneración económica atractiva, lo que podrá garantizar el sostenimiento de sus familias, recobrar la dignidad y los enrumbe a la resocialización.

El Régimen Tributario es el que con mayor frecuencia enerva la formalización de las empresas que se encuentran en situación «clandestina».

La mayoría de los tratadistas sostiene, y con razón, que de auspiciarse alguna medida tributaria de exoneración para microempresas, ello podría alentar la formación de empresas tipo «mosaico» en las organizaciones medianas ya existentes. Sin embargo, esa tendencia, que resultaría postiza y regresiva, podría estar, con relativa facilidad, sujeta a control.

Lo cierto del caso es que, por lo menos desde un punto de vista teórico e inicial, la microempresa no debería soportar tributos porque sería como obligar a un neonato a ser donante de sangre. Razón por la cual:

El régimen tributario debe ser promocional y estable. Los aportes y contribuciones de las micro y las pequeñas empresas deben ser evaluados y aquilatados por su generación de empleo, descentralización y exportaciones, antes que como cotizantes al arca fiscal.

Se propone que las microempresas no tributen, en ningún caso, durante un lapso de tres años; debiendo hacerlo, necesariamente, a partir del cuarto año, puesto que tendrían que cumplir la exigencia de convertirse en pequeñas.
Las pequeñas empresas soportarían una carga única equivalente al $5 \%$ de las ventas mensuales. También en este caso, el lapso acordado para que evolucionen a medianas será de tres años.

$>$ Las medianas empresas estarian sujetas al sistema tributario normal vigente en la actualidad; y gozarían, naturalmente, de todos los beneficios promocionales descritos anteriormente: organización, capacitación, financiamiento, asociación pro exportación, etc.

- El tributo único se declararía y pagaría mensualmente con efecto cancelatorio, en el formulario único o a través del sistema "pago fácil» instrumentado por la SUNAT.

$>$ Un Reglamento actualizado determinaría cuáles tipos de documentos (facturas, boletas, guías) estaría obligada a entregar en cada caso la micro, pequeña y mediana empresa así como en cuáles casos, eventualmente, el documento comercial podría servir de crédito fiscal o merecer el drawback.

En cuanto al Régimen Laboral concierne, las propuestas que particularmente obligarían a las micro y pequeña empresa, serían las siguientes:

Los trabajadores percibirán, por lo menos, la remuneración mínima vital.

- La jornada laboral es de ocho horas diarias y seis días a la semana.

Se reconoce el descanso semanal obligatorio y días feriados.

El derecho vacacional será de 15 días por cada año de servicio.

$>$ La única contribución obligatoria será la del seguro social de salud.

Durante su vigencia, la micro y pequeña empresa no está obligada a reconocer la estabilidad laboral. 
Tampoco está obligada a brindar liquidación por tiempo de servicios.

\section{REFERENCIAS}

ALADI. «Cómo generarpotencial exportador en las pymes». Foro virtual, 2004.

AMIAN MARTÍNEZ, Rafael. Posibles ventajas generales para las pymes. Cámara oficial de comercio e industria de Madrid, 1998.

LEVANO de ROSSI, Cecilia. Estadística de la micro y pequeña empresa. Lima-Perú, Ministerio de Trabajo y Promoción del Empleo.

ORGANIZACIÓN DE LAS NACIONES UNIDAS. Conferencia de las Naciones Unidas sobre el Comercio y Desarrollo. Marzo de 2005.
PROMPYME. «Potencial de la Pequeña Empresa como Proveedora del Estado. Junio de 2003.

SERRA PERIS, José Manuel. La PYME Industrial en el Nuevo Modelo de Crecimiento. Madrid, 1996.

VILLARAN, Fernando. Riqueza popular, pasion y gloria de la pequeña empresa. LimaPerú, Ediciones del Congreso de la República, 1998.

YACSAHUACHE MONJE, Ciro Alonso. Análisis del Financiamiento de las Pequeñas y Microempresas.

ZORRILLA, Juan Pablo. Elcrecimiento de una pequeña mediana empresa dentro del contexto capitalista. 2004.

http://www.prompyme.gob.pe http://www.mincetur.gob.pe 